\title{
Operator-valued Fourier multipliers in vector-valued function spaces and application
}

\section{Veli B. Shakhmurov}

Received: 23.03.2020 / Revised: 03.09.2020 / Accepted: 13.10.2020

\begin{abstract}
The operator-valued Fourier multiplier theorems in E-valued weighted Lebesgue and Besov spaces are studied. These results permit us to show embedding theorems in weighted Besov-Lions type spaces $B_{p, q, \gamma}^{l, s}\left(\Omega ; E_{0}, E\right)$, where $E_{0}, E$ are two Banach spaces and $E_{0} \subset E$. The most regular class of interpolation space $E_{\alpha}$, between $E_{0}$ and $E$ are found such that the mixed differential operator $D^{\alpha}$ is bounded from $B_{p, q, \gamma}^{l, s}\left(\Omega ; E_{0}, E\right)$ to $B_{p, q, \gamma}^{s}\left(\Omega ; E_{\alpha}\right)$ and Ehrling-Nirenberg-Gagliardo type sharp estimates are established. By using these results the $B_{p, q, \gamma}^{s}$-separability properties of degenerate differential operators are studied.
\end{abstract}

Keywords. Banach space-valued functions, operator-valued multipliers, embedding of abstract weighted spaces, abstract differential equations, interpolation of Banach spaces

Mathematics Subject Classification (2010): 42B15, 46N20, 35K22, 35S99

\section{Introduction}

Fourier multipliers in vector-valued function spaces has been studied e.g. in [12], [17], [28], [31]. Operator-valued Fourier multipliers in abstract function spaces have been investigated in [1], [8 - 11], [13]. Mikhlin type Fourier multipliers in scalar weighted spaces have been studied e.g. in [14] and [30]. Moreover, operator-valued Fourier multipliers in weighted abstract $L_{p}$ spaces were investigated e.g. in [2], [7], [13], [16]. Regularity properties of abstract differential equations have been studied e.g. in [1], [3], [9], [21 - 26], [30]. A comprehensive introduction to DOEs and historical references may be found in [1] and [30] .

In the paper operator-valued multiplier theorems in $E$-valued Besov space

$$
X=B_{p, q, \gamma}^{s}\left(\mathbb{R}^{n} ; E\right)
$$

are shown. Then we consider the $E$-valued anisotropic Sobolev-Besov spaces

$$
Y=B_{p, q, \gamma}^{l, s}\left(\Omega ; E_{0}, E\right),
$$

here $E_{0}, E$ are two Banach spaces, $E_{0}$ is continuously and densely embedded into $E$, and $\gamma=\gamma(x)$ is a weighted function from $A_{p}, p \in(1, \infty)$ class. We prove the

Veli B. Shakhmurov

Antalya Bilim University, Antalya, Turkey

E-mail: veli.sahmurov@gmail.com 
boundedness and compactness of embedding operators in these spaces. This result generalized and improved the results $[4, \S 9,27, \S 1.7]$ for scalar Sobolev space, the result [15] for one dimensional Sobolev-Lions spaces and the results $[22,23]$ for Hilbert-space valued class. Finally, we consider anisotropic abstract elliptic equation

$$
L u=\sum_{|\alpha: l|=1} a_{\alpha} D^{\alpha} u+A u+\sum_{|\alpha: l|<1} A_{\alpha} D^{\alpha} u=f,
$$

where $a_{\alpha}$ are complex numbers, $A, A_{\alpha}(x)$ are linear operators in a Banach space $E$. Here, $l=\left(l_{1}, l_{2}, \ldots, l_{n}\right), \alpha=\left(\alpha_{1}, \alpha_{2}, \ldots, \alpha_{n}\right), l_{k}, \alpha_{k}$ are integer numbers and $|\alpha: l|=\sum_{k=1}^{n} \frac{\alpha_{k}}{l_{k}}$.

We say that the problem (1.1) is $X$-separable, if there exists a unique solution $u \in Y$ of the problem (1.1) for all $f \in X$ and there exists a positive constant $C$ independent of $f$ such that the following coercive estimate holds

$$
\sum_{|\alpha: l| \leq 1}\left\|D^{\alpha} u\right\|_{X}+\|A u\|_{X} \leq C\|f\|_{X}
$$

The estimate (1.2) implies that if $f \in X$ and $u$ is a solution of (1.1), then all terms of the equation (1.1) belong to $X$ (i.e. all terms are separable in $X$ ).

The paper is organized as follows. In Section 2 the necessary tools from Banach space theory and some background materials are given. In Sections 3-5 the multiplier theorems in vector-valued weighted Lebesgue and Besov spaces are proved. In Section 6 by using these multiplier theorems, embedding theorems in weighted abstract Besov spaces are shown. Finally, in Section 7 the separability properties of problem (1.1) is established.

\section{Notations and background}

Let $E$ be a Banach space and $\gamma=\gamma(x), x=\left(x_{1}, x_{2}, \ldots, x_{n}\right)$ be a positive measurable function on the measurable subset $\Omega \subset \mathbb{R}^{n}$. Let $L_{p, \gamma}(\Omega ; E)$ denote the space of strongly measurable $E$-valued functions that are defined on $\Omega$ with the norm

$$
\begin{aligned}
& \|f\|_{L_{p, \gamma}}=\|f\|_{L_{p, \gamma}(\Omega ; E)}=\left(\int_{\Omega}\|f(x)\|_{E}^{p} \gamma(x) d x\right)^{\frac{1}{p}}, 1 \leq p<\infty \\
& \|f\|_{L_{\infty, \gamma}(\Omega ; E)}=\operatorname{ess}_{x \in \Omega}\|f(x)\|_{E} \gamma(x), p=\infty .
\end{aligned}
$$

For $\gamma(x) \equiv 1$, the space $L_{p, \gamma}(\Omega ; E)$ will be denoted by $L_{p}=L_{p}(\Omega ; E)$.

The weight $\gamma$ is said to be satisfy an $A_{p}$ condition [18], i.e., $\gamma \in A_{p}, 1<p<\infty$ if there is a positive constant $C$ such that

$$
\left(\frac{1}{|Q|} \int_{Q} \gamma(x) d x\right)\left(\frac{1}{|Q|} \int_{Q} \gamma^{-\frac{1}{p-1}}(x) d x\right)^{p-1} \leq C,
$$

for all rectangles $Q \subset \mathbb{R}^{n}$.

The Banach space $E$ is called a UMD-space and written as $E \in$ UMD if only if the Hilbert operator

$$
(H f)(x)=\lim _{\varepsilon \rightarrow 0} \int_{|x-y|>\varepsilon} \frac{f(y)}{x-y} d y
$$


is bounded in the space $L_{p}(\mathbb{R}, E), p \in(1, \infty)$ (see e.g. [6]). UMD spaces include e.g. $L_{p}, l_{p}$ spaces and Lorentz spaces $L_{p q}, p, q \in(1, \infty)$. Let $\mathbb{C}$ be the set of complex numbers and

$$
S_{\varphi}=\{\xi ; \xi \in \mathbb{C}, \quad|\arg \xi| \leq \varphi\} \cup\{0\}, 0 \leq \varphi<\pi .
$$

Let $E_{1}$ and $E_{2}$ be two Banach spaces. We denote that the space of linear bounded operators from $E_{1}$ to $E_{2}$ by $L\left(E_{1}, E_{2}\right)$. For $E_{1}=E_{2}=E$ it will be denoted by $L(E)$. A linear operator $A$ is said to be positive in $E$, with bound $M$ if $D(A)$ is dense on $E$ and

$$
\left\|(A+\xi I)^{-1}\right\|_{L(E)} \leq M(1+|\xi|)^{-1}
$$

with $\xi \in S_{\varphi}, \varphi \in[0, \pi)$, where $M$ is a positive constant and $I$ is an identity operator in $E$. Sometimes instead of $A+\xi I$ will be written $A+\xi$ and it is denoted by $A_{\xi}$. It is known $[28, \S 1.15 .1]$ there exist fractional powers $A^{\theta}$ of the positive operator $A$.

Definition 2.1. A positive operator $A$ is said to be $R$-positive in a Banach space $E$ if there exists $\varphi \in[0, \pi)$ such that the set

$$
\left\{(\xi)(A+\xi I)^{-1}: \xi \in S_{\varphi}\right\}
$$

is $R$-bounded (see e.g. [9]).

We denote the space of compact operators in $E$ by $\sigma_{\infty}(E)$. Let $E\left(A^{\theta}\right)$ denote the space $D\left(A^{\theta}\right)$ with the graphical norm defined as

$$
\|u\|_{E\left(A^{\theta}\right)}=\left(\|u\|^{p}+\left\|A^{\theta} u\right\|^{p}\right)^{\frac{1}{p}}, 1 \leq p<\infty,-\infty<\theta<\infty .
$$

By $\left(E_{1}, E_{2}\right)_{\theta, p}$ will be denoted interpolation spaces obtained from $\left\{E_{1}, E_{2}\right\}$ by the $K$-method [28, §1.3.1], where $\theta \in(0,1), p \in[0,1]$. We denote by $D\left(\mathbb{R}^{n} ; E\right)$ the space of $E$-valued $C^{\infty}$ - function with compact support, equipped with the usual inductive limit topology and $S(E)=S\left(\mathbb{R}^{n} ; E\right)$ denote the $E$-valued Schwartz space of rapidly decreasing, smooth functions. For $E=\mathbb{C}$ we will denoted their $D\left(\mathbb{R}^{n}\right)$ and $S=S\left(\mathbb{R}^{n}\right)$, respectively. $D^{\prime}\left(\mathbb{R}^{n} ; E\right)=L\left(D\left(\mathbb{R}^{n}\right), E\right)$ denote the space of $E$-valued distributions and $S^{\prime}(E)=S^{\prime}\left(\mathbb{R}^{n} ; E\right)$ is a space of linear continued mapping from $S\left(\mathbb{R}^{n}\right)$ into $E$. The Fourier transform for $u \in S^{\prime}\left(\mathbb{R}^{n} ; E\right)$ is defined by

$$
F(u)(\varphi)=u(F(\varphi)), \varphi \in S\left(R^{n}\right) .
$$

Let $\gamma$ be such that $S\left(\mathbb{R}^{n} ; E_{1}\right)$ is dense in $L_{p, \gamma}\left(\mathbb{R}^{n} ; E_{1}\right)$. A function $\Psi \in C^{(l)}\left(\mathbb{R}^{n} ; L\left(E_{1}, E_{2}\right)\right)$ is called a multiplier from $L_{p, \gamma}\left(\mathbb{R}^{n} ; E_{1}\right)$ to $L_{q, \gamma}\left(\mathbb{R}^{n} ; E_{2}\right)$ if there exists a positive constant $C$ such that

$$
\left\|F^{-1} \Psi(\xi) F u\right\|_{L_{q, \gamma}\left(\mathbb{R}^{n} ; E_{2}\right)} \leq C\|u\|_{L_{p, \gamma}\left(\mathbb{R}^{n} ; E_{1}\right)}
$$

for all $u \in S\left(\mathbb{R}^{n} ; E_{1}\right)$.

In a similar way, we can define the multiplier from $B_{p, q, \gamma}^{s}\left(\mathbb{R}^{n} ; E_{1}\right)$ to $B_{p, q, \gamma}^{s}\left(\mathbb{R}^{n} ; E_{2}\right)$. We denote the set of all multipliers from $B_{p, q, \gamma}^{s}\left(\mathbb{R}^{n} ; E_{1}\right)$ to $B_{p, q, \gamma}^{s}\left(\mathbb{R}^{n} ; E_{2}\right)$ by $M_{p, q, \gamma}^{s, \gamma}\left(E_{1}, E_{2}\right)$. For $E_{1}=E_{2}=E$ we denote $M_{p, p, \gamma}^{s, \gamma}\left(E_{1}, E_{2}\right)$ by $M_{p, q, \gamma}^{s, \gamma}(E)$.

Definition 2.2. Let $\gamma \in A_{q}$ for $q \in[1, \infty]$. Assume that $E$ is a Banach space and $p \in[1,2]$. Suppose that there exists a positive constant $C_{0}=C_{0}(p, \gamma, E)$ so that

$$
\|F u\|_{L_{p^{\prime}, \gamma^{\prime}}\left(\mathbb{R}^{n} ; E\right)} \leq C_{0}\|F u\|_{L_{p, \gamma}\left(\mathbb{R}^{n} ; E\right)}
$$


for $\frac{1}{p}+\frac{1}{p^{\prime}}=1, \gamma^{\prime}(\cdot)=\gamma^{-\frac{1}{p-1}}(\cdot)$ and each $u \in S\left(\mathbb{R}^{n} ; E\right)$. Then $E$ is called weighted Fourier type $\gamma$ and $p$. It is called Fourier type $p \in[1,2]$ if $\gamma(x) \equiv 1$ (see e.g [19]).

Remark 2.1. The estimate (2.1) shows that each Banach space $E$ has weighted Fourier type $\gamma$ and 1. By Bourgain [6] has shown that each $B$-convex Banach space (thus, in particular, each uniformly convex Banach space) has some non-trivial Fourier type $p \in[1,2]$, i.e. $U M D$ spaces are Fourier type for some $p \in[1,2]$.

In order to define abstract Besov spaces we consider the dyadic-like subsets $\left\{J_{k}\right\}_{k=0}^{\infty},\left\{I_{k}\right\}_{k=0}^{\infty}$ of $\mathbb{R}^{n}$ and partition of unity $\left\{\varphi_{k}\right\}_{k=0}^{\infty}$ defined e.g. in $[28, \S 2.3 .2]$.

Remark 2.2. Note the following useful properties are satisfied:

$\operatorname{supp} \varphi_{k} \subset \bar{I}_{k}$ for each $k \in \mathbb{N}_{0} ; \sum_{k=0}^{\infty} \varphi_{k}(s)=1$ for each $s \in \mathbb{R}^{n} ; I_{m} \cap \operatorname{supp} \varphi_{k}=\emptyset$ if $|m-k|>1 ; \varphi_{k-1}(s)+\varphi_{k}(s)+\varphi_{k+1}(s)=1$ for each $s \in \operatorname{supp} \varphi_{k}$ and $k \in \mathbb{N}_{0}$.

Among the many equivalent descriptions of Besov spaces, the most useful one for us is given in terms of the so called Littlewood-Paley decomposition. This means that we consider $f \in S^{\prime}(E)$ as a distributional sum $f=\sum_{k} f_{k}$ analytic functions $f_{k}$ whose Fourier transforms have support in dyadic-like $I_{k}$ and then define the Besov norm in terms of the $f_{k}$ 's.

Definition 2.3. Let $\gamma \in A_{q}, 1 \leq r, q \leq \infty$ and $s \in \mathbb{R}$. Let $l_{r}(E)$ denotes $E$-valued sequence space $[28, \S 1.18]$.

The Besov space $Y^{s}=B_{q, r, \gamma}^{s}\left(\mathbb{R}^{n} ; E\right)$ is the space of all $f \in S^{\prime}\left(\mathbb{R}^{n} ; E\right)$ for which

$$
\begin{gathered}
\|f\|_{B_{q, r, \gamma}^{s}\left(\mathbb{R}^{n} ; E\right)}=\left\|\left\{2^{k s}\left(\check{\varphi}_{k} * f\right)\right\}_{k=0}^{\infty}\right\|_{l_{r}\left(L_{q, \gamma}\left(\mathbb{R}^{n} ; E\right)\right)} \\
=\left\{\begin{array}{l}
{\left[\sum_{k=0}^{\infty} 2^{k s r}\left\|\check{\varphi}_{k} * f\right\|_{L_{q, \gamma}\left(\mathbb{R}^{n} ; E\right)}^{r}\right]^{\frac{1}{r}}<\infty, \text { if } 1 \leq r<\infty,} \\
\sup _{k \in \mathbb{N}_{0}}\left[\sum_{k=0}^{\infty} 2^{k s}\left\|\check{\varphi}_{k} * f\right\|_{L_{q, \gamma}\left(\mathbb{R}^{n} ; E\right)}\right]<\infty, \text { if } r=\infty .
\end{array}\right.
\end{gathered}
$$

$B_{q, r, \gamma}^{s}\left(\mathbb{R}^{n} ; E\right)$-together with the norm in $(2.1)$ is a Banach space. $\stackrel{\circ}{q, r, \gamma}_{E}^{s}\left(\mathbb{R}^{n} ; E\right)$ is the closure of $S\left(\mathbb{R}^{n} ; E\right)$ in $B_{q, r, \gamma}^{s}\left(\mathbb{R}^{n} ; E\right)$ with the induced norm. For $E=\mathbb{C}$ and $\gamma(x) \equiv 1$ the space $B_{q, r, \gamma}^{s}\left(\mathbb{R}^{n} ; E\right)$ states to be the usual Besov spasce (see e.g. [4], $[13])$.

Let $\Omega$ be a domain in $\mathbb{R}^{n}$. Here, $B_{q, r, \gamma}^{s}(\Omega ; E)$ denotes the space of restrictions to $\Omega$ of all functions in $B^{s}=B_{q, r, \gamma}^{s}\left(\mathbb{R}^{n} ; E\right)$ with the norm given by

$$
\|u\|_{B_{q, r, \gamma}^{s}(\Omega ; E)}=\inf _{g \in B^{s},\left.g\right|_{\Omega}=u}\|g\|_{B_{q, r, \gamma}^{s}\left(\mathbb{R}^{n} ; E\right)} .
$$

Let $l=\left(l_{1}, l_{2}, \ldots, l_{n}\right), l_{k}$ are positive integers, $s \in \mathbb{R}$ and $1 \leq q, r \leq \infty$. Here, $B_{q, r, \gamma}^{l, s}(\Omega ; E)$ denote a $E$-valued Sobolev-Besov weighted space of functions $u \in B_{q, \theta, \gamma}^{s}(\Omega ; E)$ that have weak derivatives $D_{k}^{l_{k}} u=\frac{\partial^{l_{k}}}{\partial x_{k}^{l_{k}}} u \in B_{q, r, \gamma}^{s}(\Omega ; E)$ with the norm

$$
\|u\|_{B_{q, \theta, \gamma}^{l,, s}(\Omega ; E)}=\|u\|_{B_{q, r, \gamma}^{s}(\Omega ; E)}+\sum_{k=1}^{n}\left\|D_{k}^{l_{k}} u\right\|_{B_{q, \theta r \gamma}^{s}(\Omega ; E)}<\infty .
$$


Let $E_{0}$ be continuously and densely belongs into $E . B_{q, \theta, \gamma}^{l, s}\left(\Omega ; E_{0}, E\right)$ denotes the space $B_{q, \theta, \gamma}^{s}\left(\Omega ; E_{0}\right) \cap B_{q, \theta, \gamma}^{l, s}(\Omega ; E)$ with the norm

$$
\|u\|_{B_{q, \theta, \gamma}^{l, s}}=\|u\|_{B_{q, \theta, \gamma}^{l, s}\left(\Omega ; E_{0}, E\right)}=\|u\|_{B_{q, \theta, \gamma}^{s}\left(\Omega ; E_{0}\right)}+\sum_{k=1}^{n}\left\|D_{k}^{l_{k}} u\right\|_{B_{q, \theta, \gamma}^{s}(\Omega ; E)}<\infty .
$$

Let $\left(E(X) ; E^{*}\left(X^{*}\right)\right)$ be one of the pairs. There is an embedding of $E^{*}\left(X^{*}\right) \subset$ $[E(X)]^{*}$ as a norming subspace for $E(X)$. This embedding is given by the duality map

$$
\langle., .\rangle_{E(X)}: E^{*}\left(X^{*}\right) \times E(X) \rightarrow \mathbb{C},
$$

where

$$
\langle g, f\rangle_{L_{q, \gamma}(X)}=\int_{\mathbb{R}^{n}}\langle g(t), f(t)\rangle_{X} d t=\int_{\mathbb{R}^{n}} g(t) f(t) d t
$$

in weighted Lebesgue space setting with $E=L_{q, \gamma}$ and

$$
\langle g, f\rangle_{B_{q, r, \gamma}^{s}(X)}=\sum_{n, m \in \mathbb{N}_{0}}\left\langle\check{\varphi}_{n} * g, \check{\varphi}_{m} * f\right\rangle_{L_{q, \gamma}(X)}
$$

in Besov space setting with $E=B_{q, r, \gamma}^{s}(X)$. One can check that this definition of duality is independent of the choice of the $\left\{\varphi_{k}\right\}_{k=0}^{\infty}$.

\section{The Fourier transform in weighted Besov spaces}

Let

$$
X_{q, \gamma}=L_{q, \gamma}\left(\mathbb{R}^{n} ; E\right), B_{q, r, \gamma}^{s}=B_{q, r, \gamma}^{s}(E)=B_{q, r, \gamma}^{s}\left(\mathbb{R}^{n} ; E\right) .
$$

By applying the Hausdorff-Young inequality we get the following estimates for the Fourier transform on Besov spaces

Theorem 3.1. Assume that $\gamma \in A_{p}$. Let $E$ be a Banach space with weighted Fourier type $\gamma$ and $p \in(1,2]$. Let $1 \leq q \leq p^{\prime}$ and $s \geq n\left(\frac{1}{q}-\frac{1}{p^{\prime}}\right)$ and $1 \leq r \leq \infty$. Then there exists constant $C$, depending only on $C_{0}(p, \gamma, E)$ so that if $f \in B_{q, r, \gamma}^{s}$ then

$$
\left\|\left\{\widehat{f} \chi_{J_{m}}\right\}_{m=0}^{\infty}\right\|_{l_{r}\left(X_{q,} \gamma^{\prime}\right)} \leq C\|f\|_{B_{p, r, \gamma}^{s}}
$$

where $C_{0}(p, \gamma, E)$ is a positive constant defined in the Definition 2.1 and $\gamma^{\prime}=\gamma^{-\frac{1}{p-1}}$.

An immediate corollary of Theorem 3.1 follows by choosing for $q=r=1$ and $r=q=p^{\prime}$ we obtain respectively

Corollary 3.1. Assume that $\gamma \in A_{p}$. Let $E$ be a Banach space with Fourier type $\gamma$ and $p \in(1,2]$. Then the Fourier transform $F$ defines the following bounded operator

$$
F: B_{p, 1, \gamma}^{\frac{n}{p}} \rightarrow L_{1, \gamma^{\prime}}\left(\mathbb{R}^{n} ; E\right), F: B_{p, p^{\prime}, \gamma}^{0} \rightarrow L_{p^{\prime}, \gamma^{\prime}}\left(\mathbb{R}^{n} ; E\right) .
$$

The norms of the above maps $F$ are bounded by a constant depending only on $C_{0}(n, E)$.

Theorem 3.1 and Corollary 3.1 remain valid if $F$ is replaced with $F^{-1}$. 
Proof of Theorem 3.1. Let $f \in B_{p, r, \gamma}^{s}$. Then, for each $k \in \mathbb{N}_{0}$, since $\check{\varphi}_{k} * f \in X_{q, \gamma}$ and $E$ has weighted Fourier type $\gamma$ and $p$,

$$
\varphi_{k} \cdot \widehat{f}=F\left(\check{\varphi}_{k} * f\right) \in X_{p^{\prime}, \gamma^{\prime}} .
$$

Thus by Remark 2.2,

$$
\widehat{f} \chi_{J_{m}}=\left(\sum_{k=m-1}^{m+1} \varphi_{k} \cdot \widehat{f}\right) \chi_{J_{m}} \in X_{q, \gamma^{\prime}} \text { for each } m \in \mathbb{N}_{0} .
$$

Moreover, by Definition 2.2 we get

$$
\left\|\varphi_{k} \widehat{f}\right\|_{X_{p^{\prime}, \gamma^{\prime}}}=\left\|F\left(\check{\varphi}_{k} * f\right)\right\|_{X_{p^{\prime}, \gamma^{\prime}}} \leq C_{0}\left\|\check{\varphi}_{k} * f\right\|_{X_{p, \gamma}},
$$

i.e.

$$
\sum_{k=m-1}^{m+1} 2^{k s}\left\|\varphi_{k} \widehat{f}\right\|_{X_{p^{\prime}, \gamma^{\prime}}} \leq C_{0} \sum_{k=m-1}^{m+1} 2^{k s}\left\|\check{\varphi}_{k} * f\right\|_{X_{p, \gamma}} .
$$

In view of (3.4), it suffices to show that there exists the positive constant $C_{1}$ so that the following holds

$$
\left\|\widehat{f} \chi_{J_{m}}\right\|_{X_{q, \gamma^{\prime}}} \leq C_{1} \sum_{k=m-1}^{m+1} 2^{k s}\left\|\varphi_{k} \widehat{f}\right\|_{X_{p^{\prime}, \gamma^{\prime}}} .
$$

We consider the case when $q \neq p^{\prime}$. Choose $1 \leq \sigma<p$, that $\frac{1}{q}=\frac{1}{p^{\prime}}+\frac{1}{\sigma}$; so, $\frac{n}{\sigma} \leq s$. By the generalized Hölder's inequality for each $m \in \mathbb{N}_{0}$,

$$
\begin{aligned}
& \left\|\widehat{f} \chi_{J_{m}}\right\|_{X_{q, \gamma^{\prime}}} \leq \sum_{k=m-1}^{m+1}\left\|\varphi_{k} \widehat{f} \chi_{J_{m}}\right\|_{L_{q, \gamma}\left(J_{m} ; E\right)} \\
& \leq \sum_{k=m-1}^{m+1}\left\|\varphi_{k}\left(\frac{1+|\cdot|}{4}\right)^{\frac{n}{\sigma}} \widehat{f} \gamma^{\frac{1}{p^{\prime}}}(\cdot)\right\|_{L_{p^{\prime}, \gamma^{\prime}}\left(J_{m} ; E\right)}\left\|\gamma^{\frac{1}{p}}\left(\frac{1+|\cdot|}{4}\right)^{-\frac{n}{\sigma}}\right\|_{L_{\sigma}\left(J_{m}\right)} \\
& \leq \sum_{k=m-1}^{m+1}\left\|\widehat{f} \varphi_{k}\right\|_{L_{p^{\prime}, \gamma^{\prime}}\left(J_{m} ; E\right)}\left\|\left(\frac{1+|\cdot|}{4}\right)^{\frac{n}{\sigma}} \chi_{J_{m}}\right\|_{L_{\infty}} \leq C_{2} \sum_{k=m-1}^{m+1} 2^{k s}\left\|\widehat{f} \varphi_{k}\right\|_{L_{p^{\prime}, \gamma^{\prime}}\left(J_{m} ; E\right)},
\end{aligned}
$$

where $C_{2}$ is a positive constant defined by

$$
\begin{gathered}
C_{2}=\left\|\gamma^{\frac{1}{p}}\left(\frac{1+|\cdot|}{4}\right)^{-\frac{n}{\sigma}}\right\|_{L_{\sigma}\left(J_{m}\right)} \leq\left\|\left(\frac{1+|\cdot|}{4}\right)^{-n}\right\|_{L_{\infty}\left(J_{m}\right)}\left\|\gamma^{\frac{\sigma}{p}}\right\|_{L\left(J_{m}\right)} \\
\leq 4^{n}\left[\sup _{m \in \mathbb{N}_{0}} 2^{-(m-1) n} \int_{J_{m}} \gamma^{\frac{\sigma}{p}}(s) d s\right]^{\frac{1}{\sigma}} .
\end{gathered}
$$

In view of $\gamma \in A_{p}$, we have

$$
\sup _{m \in \mathbb{N}_{0}} 2^{-(m-1) n} \int_{J_{m}} \gamma^{\frac{\sigma}{p}}(s) d s<\infty .
$$


For $q=p^{\prime}$ and for each $m \in \mathbb{N}$ we get

$$
\begin{aligned}
\left\|\widehat{f} \chi_{J_{m}}\right\|_{X_{q, \gamma}} & \leq \sum_{k=m-1}^{m+1}\left\|\varphi_{k} \widehat{f} \chi_{J_{m}}\right\|_{L_{p^{\prime}, \gamma^{\prime}}\left(J_{m} ; E\right)} \\
& \leq \sum_{k=m-1}^{m+1} 2^{k s}\left\|\varphi_{k} \widehat{f}\right\|_{X_{p^{\prime}, \gamma^{\prime}}} .
\end{aligned}
$$

So, from (3.6)-(3.8) we obtain (3.5) .

Remark 3.1. By using the embedding $W_{p, \gamma}^{j}\left(\mathbb{R}^{n} ; E\right) \subset B_{q, r, \gamma}^{s}$ for $s<j \in \mathbb{N}$ we get that the statement of Theorem 3.1 remains valid if $B_{q, r, \gamma}^{s}$ is replaced by $W_{p, \gamma}^{j}\left(\mathbb{R}^{n} ; E\right)$.

Also, it follows from Corollary 3.1 that if $E$ has weighted Fourier type for $\gamma \in A_{\nu}$, $\nu \in[1, \infty]$ and $j>\frac{n}{p}$ then the Fourier transform $F$ defines bounded operator:

$$
W_{p, \gamma}^{j}\left(\mathbb{R}^{n} ; E\right) \rightarrow X_{1, \gamma^{\prime}} .
$$

Furthermore, if $E$ has weighted Fourier type for $\gamma \in A_{\nu}, \nu \in[1, \infty]$ and $j>\frac{n}{p}$ then there is a constant $C$ so that

$$
\|\hat{f}\|_{X_{1, \gamma^{\prime}}} \leq C\|f\|_{X_{p, \gamma}}^{1-\frac{n}{j p}}\left[\sum_{|\alpha|=j}\left\|D^{\alpha} f\right\|_{X_{p, \gamma}}\right]^{\frac{n}{j p}}
$$

for each $f \in W_{p, \gamma}^{j}\left(\mathbb{R}^{n} ; E\right)$.

\section{Fourier multipliers on weighted Lebesgue spaces}

Let $m: \mathbb{R}^{n} \rightarrow L\left(E_{1}, E_{2}\right)$ be a bounded measurable function. In this section, we identify conditions on $m$, generalizing the classical Mikhlin condition so that the multiplication operator induced by $m$, i.e. the operator: $u \rightarrow T_{m}=F^{-1} m F u$ is bounded from $L_{q, \gamma}\left(\mathbb{R}^{n} ; E_{1}\right)$ to $L_{q, \gamma}\left(\mathbb{R}^{n} ; E_{2}\right)$. We will first give rather general criteria for Fourier multipliers in terms of the weighted Besov norm of the multiplier function; later we derive from these results analogues of the classical Mikhlin and Hörmander conditions. To simplify the statements of our results, we let

$$
M_{p, \gamma}(m)=\inf _{a>0}\left\{\|m(a, .)\|_{B_{p, 1 . \gamma}^{\frac{n}{p}}\left(\mathbb{R}^{n} ; L\left(E_{1}, E_{2}\right)\right)}\right\} .
$$

Let

$$
\begin{gathered}
X_{k}=X_{q}\left(E_{k}\right)=L_{q, \gamma}\left(\mathbb{R}^{n} ; E_{k}\right), k=1,2, \\
X_{1}(L)=L_{1}\left(\mathbb{R}^{n} ; L\left(E_{1}, E_{2}\right)\right), Y=B_{p, 1 . \gamma}^{\frac{n}{p}}\left(\mathbb{R}^{n} ; L\left(E_{1}, E_{2}\right)\right) .
\end{gathered}
$$

First we give a multiplier result from $X_{1}$ to $X_{2}$ in the spirit of Steklin's theorem.

Theorem 4.1. Assume that $\gamma \in A_{q}$ for $q \in[1, \infty]$. Let $E_{1}, E_{2}$ be Banach spaces with weighted Fourier type $\gamma$ and $p \in p \in(1,2]$. Suppose that

$$
T_{m} \in B_{p, 1, \gamma}^{\frac{n}{p}}\left(\mathbb{R}^{n} ; L\left(X_{1}, X_{2}\right)\right) .
$$


Then there exists a constant $C$ depending only on $C_{01}\left(p, \gamma, E_{1}\right)$ and $C_{02}\left(p, \gamma, E_{2}\right)$, so that if $m \in Y$, then $m$ is a Fourier multiplier from $X_{1}$ to $X_{2}$ and

$$
\left\|T_{m}\right\|_{L\left(X_{1}, X_{2}\right)} \leq C M_{p, \gamma}(m)
$$

for each $q \in[1, \infty]$. $A$.

Let $E^{*}$ denotes the dual space of $E$ and $A^{*}$ denotes the conjugate of the operator

The proof of Theorem 4.1 uses the following lemma.

Lemma 4.1. Assume that $\gamma \in A_{q}$ for $q \in[1, \infty]$ and $k \in X_{1}(L)$. Suppose that there exists constants $C_{i}$ so that for each $x \in E_{1}$ and $x^{*} \in E_{2}^{*}$

$$
\int_{\mathbb{R}^{n}}\|k(s) x\|_{E_{2}} d s \leq M_{0}\|x\|_{E_{1}}, \quad \int_{\mathbb{R}^{n}}\left\|k^{*}(s) x^{*}\right\|_{E_{1}^{*}} d s \leq M_{1}\left\|x^{*}\right\|_{E_{2}^{*}} .
$$

Then the convolution operator $K: X_{1} \rightarrow X_{2}$ defined by

$$
(K f)(t)=\int_{\mathbb{R}^{n}} k(t-s) f(s) d s \text { for } t \in \mathbb{R}^{n}
$$

satisfies that

$$
\|K\|_{L\left(X_{1}, X_{2}\right)} \leq M_{0}^{\frac{1}{q}} M_{1}^{1-\frac{1}{q}} .
$$

Proof. Since $k \in X_{1}(L)$ it is well-known that (4.2) defines a bounded operator on $X_{1}$. Indeed, for $f \in X_{1} \cap X_{\infty}\left(E_{1}\right)$ we have

$$
\int_{\mathbb{R}^{n}}\|k(t-s) f(s)\|_{E_{2}} d s=\int_{\mathbb{R}^{n}}\left\|k(s) f_{s}(t)\right\|_{E_{2}} d s \leq\|k\|_{X_{1}(L)}\|f\|_{X_{\infty}\left(E_{1}\right)}
$$

for each $t \in \mathbb{R}^{n}$ and $f_{s}(t)=f(t-s)$. From (4.3) by applying the Minkowski's inequality for integral with weight $[20, \S$ A.1] we get

$$
\begin{gathered}
\|K f(\cdot)\|_{X_{2}} \leq \int_{\mathbb{R}^{n}}\left\|k(s) f_{s}(t)\right\|_{X_{2}} d s \leq \int_{\mathbb{R}^{n}}\|k(s)\|_{L\left(E_{1}, E_{2}\right)}\left\|f_{s}\right\|_{X_{1}} d s \\
=\|k\|_{X_{1}(L)}\left\|f_{s}\right\|_{X_{1}} .
\end{gathered}
$$

Now, for $q=1$ we have from (4.1)

$$
\begin{gathered}
\|K f\|_{X_{1}\left(E_{1}\right)} \leq \int_{\mathbb{R}^{n}}\left(\int_{\mathbb{R}^{n}}\left\|k(s) f_{s}(t)\right\|_{E_{1}} d s\right) \gamma(t) d t \\
\leq M_{0} \int_{\mathbb{R}^{n}}\|f(t)\|_{E_{1}} \gamma(t) d t=M_{0}\|f\|_{X_{1}\left(E_{1}\right)} .
\end{gathered}
$$

Hence,

$$
\|K\|_{L\left(X_{1}\left(E_{1}\right)\right)} \leq M_{0} .
$$

If $q=\infty$, then for each $X_{\infty}\left(E_{1}\right), x^{*} \in E_{2}^{*}$ and $t \in \mathbb{R}^{n}$ by using (4.1) we get

$$
\left|\left\langle x^{*},(K f)(t)\right\rangle_{E_{2}}\right| \leq \int_{\mathbb{R}^{n}}\left|\left\langle k^{*}(t-s) x^{*}, f(s)\right\rangle_{E_{1}}\right| \gamma(s) d s
$$




$$
\leq \int_{\mathbb{R}^{n}}\left\|k^{*}(t-s) x^{*}\right\|_{E_{1}^{*}}\|f(s)\|_{E_{1}} \gamma(s) d s \leq M_{1}\left\|x^{*}\right\|_{E_{1}^{*}}\|f\|_{X_{1}\left(E_{1}\right)} .
$$

Thus,

$$
\|K\|_{L\left(X_{1}\left(E_{1}\right)\right)} \leq M_{1}
$$

Let $\bar{X}_{\infty}\left(E_{1}\right)$ denotes the closure in $X_{\infty}\left(E_{1}\right)$ norm of the simple functions $\sum_{k=1}^{m} x_{k} \chi_{A_{k}}$, where $x_{k} \in E_{1}$, vol $A_{k}<\infty$ and $m \in \mathbb{N}$. Then one can check that $K$ maps $\bar{X}_{\infty}\left(E_{1}\right)$ into $\bar{X}_{\infty}\left(E_{1}\right)$. Indeed, for $f=\chi_{A}$, we have

$$
K f(t)=\int_{t-A} k(s) x d s \rightarrow 0 \text { for } t \rightarrow \infty
$$

and $K f$ is a continuous function from $\mathbb{R}^{n}$ to $E_{2}$. Now, the Riesz-Thorin theorem (cf. [5, Thm 5.1.2]) yields the claim for $1<p<\infty$.

Proof of Theorem 4.1. First assume in addition that $m \in S\left(L\left(E_{1}, E_{2}\right)\right)$. Hence, $\check{m} \in S\left(L\left(E_{1}, E_{2}\right)\right)$. Fix $x \in E_{1}$. For an appropriate choice of $a>0$, we can apply Corollary 3.1 to the function $t \rightarrow m(a t) x$ in $B_{p, 1 . \gamma}^{\frac{n}{p}}\left(\mathbb{R}^{n} ; E_{2}\right)$ and use that

$$
F^{-1}[m(a .) x](s)=a^{-n} \check{m}\left(\frac{s}{a}\right) x
$$

to get

$$
\begin{gathered}
\|\check{m}(\cdot) x\|_{X_{1}\left(E_{1}\right)}=\left\|F^{-1} m(a .) x\right\|_{X_{1}\left(E_{1}\right)} \\
\leq C_{1}\|m(a .) x\|_{Y}\|x\|_{E_{1}} \leq 2 C_{1} M_{p, \gamma}\|x\|_{E_{1}},
\end{gathered}
$$

for some constant $C_{1}$ which depends on $C_{0}\left(p, \gamma, E_{2}\right)$.

By the additional assumption on $m$ we get

$$
m^{*}(\cdot) \in S\left(L\left(E_{2}^{*}, E_{1}^{*}\right)\right) \text {, and } F^{-1} m^{*}(\cdot)=[\check{m}(\cdot)]^{*} \in S\left(L\left(E_{2}^{*}, E_{1}^{*}\right)\right) .
$$

Let $x^{*} \in E_{2}^{*}$. Similarly, by applying Corollary 3.1 to an appropriate function

$$
t \rightarrow[m(a t)]^{*} x^{*} \text { in } B_{p, 1 . \gamma}^{\frac{n}{p}}\left(R^{n} ; E_{1}^{*}\right)
$$

and using the fact that $M_{p, \gamma}(m)=M_{p, \gamma}\left(m^{*}\right)$, one has

$$
\left\|[\check{m}(\cdot)]^{*} x^{*}\right\|_{X_{1}\left(E_{1}^{*}\right)} \leq 2 C_{2} M_{p, \gamma}(m)\left\|x^{*}\right\|_{E_{2}^{*}}
$$

for some constant $C_{2}$ which depends $C_{0}\left(p, \gamma, E_{1}^{*}\right)$. By Lemma 4.1, the convolution operator

$$
\left(T_{m} f\right)(t)=\int_{\mathbb{R}^{n}} \check{m}(t-s) f(s) d s
$$

satisfies

$$
\left\|T_{m}\right\|_{B\left(X_{1}, X_{2}\right)} \leq C M_{p, \gamma}(m),
$$

where $C=2 \max \left\{C_{1}, C_{2}\right\}$. Furthermore, since $m \in L_{1}\left(\mathbb{R}^{n} ; L\left(E_{1}, E_{2}\right)\right)$, then $T_{m}$ satisfies the following

$$
T_{m} f=F^{-1} m(\cdot) f(\cdot) \text { for all } f \in S\left(\mathbb{R}^{n} ; E_{1}\right),
$$

also

$$
T_{m} \in C\left(\sigma\left(X_{1}, X_{1}^{*}\right), \sigma\left(X_{2}, X_{2}^{*}\right)\right),
$$


where $\sigma\left(X_{k}, X_{k}^{*}\right)$ denote the interpolation spaces of $X_{k}, X_{k}^{*}$.

For the general case, let $m \in Y$. It is known that $S\left(\mathbb{R}^{n} ; L\left(E_{1}, E_{2}\right)\right)$ is dense in $Y$ when $\gamma \in A_{\nu}, \nu \in[1, \infty]$. Now, let we choose a sequence $\left\{m_{n}\right\}_{n}^{\infty} \subset S\left(\mathbb{R}^{n} ; L\left(E_{1}, E_{2}\right)\right)$ that converges to $m$ in the $Y$-norm and obtain operators $T_{m_{n}} \in L\left(X_{1}, X_{2}\right)$, where

$$
T_{m_{n}} f=F^{-1} m_{n}(\cdot) f(\cdot), f \in X_{1} .
$$

It is clear to see that, the properties (4.6) and (4.7) pass from $T_{m_{n}}$ to $T_{m}$. One also has that

$$
\left\|T_{m}\right\|_{L\left(X_{1}, X_{2}\right)} \leq C\|m\|_{Y} .
$$

Fix $a>0$ such that $m(a$. $) \in Y$. Then $I_{E_{2}} \circ T_{m(a .)}=T_{m} \circ I_{E_{1}}$, where $I_{\mathbb{Z}}$ : $L_{q, \gamma}\left(\mathbb{R}^{n} ; \mathbb{Z}\right) \rightarrow L_{q, \gamma}\left(\mathbb{R}^{n} ; \mathbb{Z}\right)$ is the isometry

$$
T(f)(t)=a^{\frac{n}{q}} f(a t) .
$$

Thus,

$$
\left\|T_{m}\right\|_{L\left(X_{1}, X_{2}\right)}=\left\|T_{m(a \cdot)}\right\|_{L\left(X_{1}, X_{2}\right)} \leq C\|m\|_{Y},
$$

i.e.

$$
\left\|T_{m}\right\|_{L\left(X_{1}, X_{2}\right)} \leq C M_{p, \gamma}(m)
$$

The following remark collects some basic facts about the Fourier multiplier operators $T_{m}$ given in Theorem 4.1 that will be used in the proof of Theorem 4.2.

Remark 4.1. Let $f \in X_{1}$ and let $\Omega$ be a closed subset of $\mathbb{R}^{n}$. Then the following are valid:

(a) Viewing $f$ and $T_{m} f$ as distributions, if supp $\widehat{f} \subset \Omega$ then supp $F\left(T_{m} f\right) \subset \Omega$;

(b) $T_{m_{1}+m_{2}}=T_{m_{1}}+T_{m_{2}}$. If $\varphi \in S$, then $\check{\varphi} * T_{m} f=T_{m}(\check{\varphi} * f)=T_{\varphi m}(f)$;

(c) If $\varphi \in S$ is 1 on supp $\widehat{f}$, then $T_{\varphi m}(f)=T_{m}(f)$;

(d) $T_{m}^{*}$ restricted to $L_{q^{*}, \gamma}\left(\mathbb{R}^{n} ; E_{2}^{*}\right)$ is $T_{m^{*}(-.)}$.

\section{Fourier multipliers on weighted Besov spaces}

Let $m: \mathbb{R}^{n} \rightarrow L\left(E_{1}, E_{2}\right)$ be a bounded measurable function. In this section we identify conditions on $m$, generalizing the classical Mikhlin condition so that the multiplication operator induced by $m$, i.e. the operator: $u \rightarrow T_{m}=F^{-1} m F u$ is bounded from $B^{s}\left(E_{1}\right)$ to $B^{s}\left(E_{2}\right)$. By applying this Theorem 4.1 to the blocks of the Littlewood-Paley decomposition of Besov spaces we will now get the main result of this section. Let

$$
Y_{k}\left(E_{k}\right)=B^{s}\left(E_{k}\right)=B_{q, r, \gamma}^{s}\left(\mathbb{R}^{n} ; E_{k}\right), k=1,2 .
$$

Theorem 5.1. Assume that $\gamma \in A_{q}$ for $q \in[1, \infty]$. Let $E_{1}, E_{2}$ be a Banach spaces with weighted Fourier type $\gamma$ and $p \in(1,2]$. Then there is a constant $C$ depending only on $C_{01}\left(p, \gamma, E_{1}\right)$ and $C_{02}\left(p, \gamma, E_{2}\right)$, so that if

$$
\varphi_{k} m \in B_{p, 1, \gamma}^{\frac{n}{p}}\left(\mathbb{R}^{n} ; L\left(X_{1}, X_{2}\right)\right) \text { and } M_{p, \gamma}\left(\varphi_{k} m\right) \leq A \text { for each } k \in \mathbb{N}_{0}
$$

then $m$ is a Fourier multiplier from $Y_{1}$ to $Y_{2}$ and $\left\|T_{m}\right\|_{B\left(Y_{1}, Y_{2}\right)} \leq C A$ for each $s \in \mathbb{R}$ and $q, r \in[1, \infty]$. 
Proof. By properties of $\left\{\varphi_{k}\right\}$ we have

$$
\begin{aligned}
T_{m} f=F^{-1} m \widehat{f} & =\sum_{k \in \mathbb{N}_{0}} F^{-1}\left[\left(\varphi_{k-1}+\varphi_{k}+\varphi_{k+1}\right) m F\left[\left(\check{\varphi}_{k} * f\right)\right]\right] \\
& =\sum_{k \in \mathbb{N}_{0}} T_{\left(\varphi_{k-1}+\varphi_{k}+\varphi_{k+1}\right) m}\left(\check{\varphi}_{k} * f\right)
\end{aligned}
$$

where $T_{m}$ is the Fourier multiplier operator on $X_{1}$ given by Theorem 4.1. From Theorem 4.1 implies that $m \varphi_{k}$ induces a Fourier multiplier operator $T_{m . \varphi_{k}}$ with

$$
\left\|T_{m \cdot \varphi_{k}}\right\|_{L\left(X_{1}, X_{2}\right)} \leq C M_{p, \gamma}\left(\varphi_{k} m\right) \leq C A
$$

for some constant $C$ depending only on $C_{0,1}\left(p, \gamma, E_{1}\right)$ and $C_{0,2}\left(p, \gamma, E_{2}\right)$. Let

$$
\psi_{k}=\varphi_{k-1}+\varphi_{k}+\varphi_{k+1} \text {. }
$$

Note that $\psi_{k}(s) \equiv 1$ when $s \in \operatorname{supp} \varphi_{k}$. Then $m \psi_{k}$ induces the Fourier multiplier operator $T_{m . \psi_{k}}$ with

$$
T_{m \psi_{k}}=T_{m \varphi_{k-1}}+T_{m \varphi_{k}}+T_{m \varphi_{k+1}} \in B\left(X_{1}, X_{2}\right)
$$

and

$$
\left\|T_{m . \psi_{k}}\right\|_{B\left(X_{1}, X_{2}\right)} \leq 3 C A .
$$

Define $T_{0}: S\left(E_{1}\right) \rightarrow S^{\prime}\left(E_{1}\right)$ by

$$
T_{0} f=F^{-1} m(\cdot) F f(\cdot) .
$$

If $f \in S\left(E_{1}\right)$, then $\check{\varphi}_{k} * T_{0} f=T_{m \psi_{k}}\left(\check{\varphi}_{k} * f\right)$ for each $k \in \mathbb{N}_{0}$ since

$$
\begin{gathered}
F\left[T_{m \psi_{k}}\left(\check{\varphi}_{k} * f\right)\right](\cdot)=m(\cdot) \psi_{k}(\cdot) F\left[\left(\check{\varphi}_{k} * f\right)(\cdot)\right] \\
=\varphi_{k}(\cdot) m(\cdot) \hat{f}(\cdot)=\varphi_{k}(\cdot) F\left(T_{0} f\right)=F\left[\left(\check{\varphi}_{k} * T_{0} f\right)(\cdot)\right] .
\end{gathered}
$$

So, by definition of the Besov norm

$$
\left\|T_{0} f\right\|_{Y_{2}} \leq 3 C A\left\|T_{0} f\right\|_{Y_{1}} .
$$

Thus $T_{0}$ extends to a bounded linear operator from $\stackrel{\circ}{B_{q, r, \gamma}^{s}}\left(\mathbb{R}^{n} ; E_{1}\right)$ into

$$
\stackrel{\circ}{B}_{q, r, \gamma}^{s}\left(\mathbb{R}^{n} ; E_{2}\right) .
$$

If $q, r<\infty$ then all that would remain is to verify the weak continuity condition (4.7). However, we continue with the proof in order to also cover the case $q=\infty$ or $r=\infty$. We shall show that the operator $T_{m}: Y_{1} \rightarrow Y_{2}$ defined by

$$
T_{m} f=\sum_{k=1}^{\infty} f_{k}, f_{k}=T_{m \psi_{k}}\left(\check{\varphi}_{k} * f\right) \in X_{2}
$$

is indeed a (norm) continuous operator. Fix $f \in Y_{1}$. First, we show that the formal series (5.3) defines an element in $S^{\prime}\left(E_{2}\right)$. Towards this, fix $\varphi \in S$. From Remark 4.1 implies that supp $f_{k} \subset \bar{I}_{k}$. Thus

$$
f_{k}(\varphi)=\widehat{f}_{k}(\check{\varphi})=\hat{f}_{k}\left(\psi_{k}(-\cdot) \check{\varphi}\right)=f_{k}\left(\psi_{k} * \varphi\right)
$$


and so by using Hölder inequality with weight $\gamma \in A_{q}$ as in (3.7) we get

$$
\begin{gathered}
\sum_{k=1}^{\infty}\left\|f_{k}(\varphi)\right\|_{E_{2}} \leq \sum_{k=1}^{\infty}\left\|f_{k}\right\|_{X_{2}}\left\|\gamma^{-\frac{1}{q}}\left(\psi_{k} * \varphi\right)\right\|_{L_{q^{\prime}}(\mathbb{C})} \\
\leq M \sum_{k=1}^{\infty} 2^{k s}\left\|\check{\varphi}_{k} * f\right\|_{X_{2}}\left\|2^{-k s} \psi_{k} * \varphi\right\|_{L_{q^{\prime}, \sigma}(\mathbb{C})} \leq M 2^{|s|}\|f\|_{Y_{2}}\|\varphi\|_{B_{q^{\prime}, r^{\prime}, \sigma}^{-s}}(\mathbb{C}),
\end{gathered}
$$

where

$$
\sigma(\cdot)=\gamma^{1-q}(\cdot) .
$$

Thus $\left(T_{m} f\right)(\varphi)$ for $\varphi \in S$ defines a linear map from $S$ into $E_{2}$ which is continuous by well known inclusion

$$
S\left(E_{2}\right) \subset Y_{2} \subset S^{\prime}\left(E_{2}\right) .
$$

By Remark 4.1, for each $j, k \in \mathbb{N}_{0}$

$$
\check{\varphi}_{j} * T_{m \psi_{k}}\left(\check{\varphi}_{k} * f\right)=T_{m \psi_{k}}\left(\check{\varphi}_{j} * \check{\varphi}_{k} * f\right)=\check{\varphi}_{k} * T_{m \psi_{k}}\left(\check{\varphi}_{j} * f\right) \text {. }
$$

Since the support of $\varphi_{k}$ intersects the support of $\varphi_{j}$ only for $|k-j| \leq 1$, applying Remark 4.1 further gives

$$
\begin{aligned}
\check{\varphi}_{k} * T_{m} f & =\sum_{j=k-1}^{k+1} \check{\varphi}_{k} * T_{m \psi_{j}}\left(\check{\varphi}_{j} * f\right)=\sum_{j=k-1}^{k+1} \check{\varphi}_{j} * T_{m \psi_{j}}\left(\check{\varphi}_{k} * f\right) \\
& =\sum_{j=k-1}^{k+1} T_{m \varphi_{j} \psi_{j}}\left(\check{\varphi}_{k} * f\right)=T_{m \psi_{k}}\left(\check{\varphi}_{k} * f\right) .
\end{aligned}
$$

Hence, $\check{\varphi}_{k} * T_{m} f \in X_{2}$ and

$$
\left\|\check{\varphi}_{k} * T_{m} f\right\|_{X_{2}} \leq 3 C A\left\|\check{\varphi}_{k} * f\right\|_{X_{1}},
$$

from which and in view of (5.2) it follows that range of $T_{m}$ is contained in $Y_{1}$ and that norm of $T_{m}$ as an operator from $Y_{1}$ to $Y_{2}$ is bounded by a constant depending on the items claimed. Furthermore, $T_{m}$ extends $T_{0}$; indeed, if $f \in S\left(E_{1}\right)$ then

$$
\begin{gathered}
F\left(T_{m} f\right)=\sum_{k=1}^{\infty} F\left[T_{m \psi_{k}}\left(\check{\varphi}_{k} * f\right)\right]=\sum_{k=1}^{\infty} m \psi_{k} \varphi_{k} \widehat{f} \\
=\sum_{k=1}^{\infty} m \varphi_{k} \widehat{f}=F\left(T_{0} f\right) .
\end{gathered}
$$

It remains to show only that $T_{m}$ satisfies (4.7). Since $[m(-.)]^{*}: \mathbb{R}^{n} \rightarrow L\left(Y_{2}^{*} ; Y_{1}^{*}\right)$ also satisfies condition (5.1), the Fourier multiplier operator $T_{m^{*}(-.)}$, defined by (4.6), extends to $T_{m^{*}(-)} \in L\left(Y_{2}^{*} ; Y_{1}^{*}\right)$. It suffices to show that $T_{m}^{*}$ restricted to $Y_{2}^{*}$ is $T_{m^{*}(-.)}$. Hence, fix $g \in Y_{2}^{*}, f \in B^{s}\left(E_{1}\right)$ and by using (5.4) and (2.3) we have

$$
\begin{gathered}
\left\langle T_{m}^{*} g, f\right\rangle_{Y_{1}}=\sum_{n, k \in \mathbb{N}_{0}}\left\langle\check{\varphi}_{n} * g, \check{\varphi}_{k} * T_{m} f\right\rangle_{L_{q, \gamma}\left(E_{2}\right)} \\
=\sum_{n, k \in \mathbb{N}_{0}}\left\langle\check{\varphi}_{n} * g, T_{m \psi_{k}}\left(\check{\varphi}_{k} * f\right)\right\rangle_{L_{q, \gamma}\left(E_{2}\right)} .
\end{gathered}
$$


and

$$
\begin{gathered}
\left\langle T_{m^{*}(-.)} g, f\right\rangle_{Y_{1}}=\sum_{n, k \in \mathbb{N}_{0}}\left\langle\check{\varphi}_{n} * T_{m^{*}(-.)} g, \check{\varphi}_{k} * f\right\rangle_{L_{q, \gamma}\left(E_{1}\right)} \\
=\sum_{n, k \in \mathbb{N}_{0}}\left\langle T_{m^{*}(-.) \psi_{n}(.)}\left(\check{\varphi}_{n} * g\right), \check{\varphi}_{k} * f\right\rangle_{L_{q, \gamma}\left(E_{1}\right)} .
\end{gathered}
$$

Fix $K_{0} \subset \mathbb{N}_{0}$ and choose a radial $\psi \in S$ with compact support such that $\psi$ is 1 $K_{0}+1$

on $\bigcup_{k=1}^{K_{0}+1} \operatorname{supp} \varphi_{k}$. If $n, k \in\left\{0,1, \ldots, K_{0}\right\}$, then by Remark 4.1 we get

$$
T_{m \psi_{k}}\left(\check{\varphi}_{k} * f\right)=T_{m \psi \psi_{k}}\left(\check{\varphi}_{k} * f\right)=T_{m \psi}\left(\check{\varphi}_{k} * f\right)
$$

and

$$
T_{m^{*}(-.) \psi_{n}(\cdot)}\left(\check{\varphi}_{n} * f\right)=T_{m^{*}(-.) \psi(\cdot) \psi_{n}(\cdot)}\left(\check{\varphi}_{n} * f\right)=T_{m^{*}(-.) \psi_{n}(.)}\left(\check{\varphi}_{n} * f\right) .
$$

since $m \psi$ and $m^{*}(-.) \psi_{n}(\cdot)$ satisfy the assumptions of Theorem 4.1. Hence, by (5.5)(5.8) and by Remark 4.1 we have

$$
\left\langle T_{m}^{*} g, f\right\rangle=\left\langle T_{m^{*}(-.)} g, f\right\rangle .
$$

The next lemma gives a convenient way to verify the assumption of Theorem 4.8 in terms of derivatives.

By reasoning as Lemma 4.10 and Corollary 4.11 in [11] we obtain

Lemma 5.1. Let $\frac{n}{p}<l \in \mathbb{N}$ and $\sigma \in[p, \infty]$. If $m \in C^{l}\left(\mathbb{R}^{n} ; L\left(E_{1}, E_{2}\right)\right)$ and there exists a positive constant $A$ so that

$$
\left\|D^{\alpha} m\right\|_{L_{\sigma}\left(\mathbb{R}^{n} ; L\left(E_{1}, E_{2}\right)\right)} \leq A
$$

for each $k \in \mathbb{N}, \alpha \in \mathbb{N}_{0}^{n}$ with $|\alpha| \leq l-1$. Then $m$ satisfies condition (5.1) of Theorem 5.1 .

Corollary 5.1. Let $q, r \in[1, \infty]$ and $s \in \mathbb{R}$. If $m \in C^{l}\left(\mathbb{R}^{n} ; L\left(E_{1}, E_{2}\right)\right)$ and there exists a positive constant $A$ so that

$$
\sup _{x \in \mathbb{R}^{n}}(1+|x|)^{|\alpha|}\left\|D^{\alpha} m\right\|_{L_{\sigma}\left(\mathbb{R}^{n} ; L\left(E_{1}, E_{2}\right)\right)} \leq A
$$

for each $k \in \mathbb{N}, \alpha \in \mathbb{N}_{0}^{n}$ with $|\alpha| \leq l$ and $m_{k}(\cdot)=m\left(2^{k-1}\right.$.). Then $m$ is a Fourier multiplier from $Y_{1}$ to $Y_{2}$ provided one of the following conditions hold:

(a) $E_{1}$ and $E_{2}$ are arbitrary Banach spaces and $l=n+1$;

(b) $E_{1}$ and $E_{2}$ are uniformly convex Banach spaces and $l=n$;

(c) $E_{1}$ and $E_{2}$ have Fourier type $p$ and $l=\left[\frac{n}{p}\right]+1$.

\section{Embedding theorems in Besov-Lions type spaces}

In this section embedding theorems in abstract Besov spaces in terms of interpolation of Banach spaces are derived. Note, that embedding of function spaces were studied e.g. in $[1,2,4],[12]$. Embedding in abstract function spaces in terms of interpolation were studied e.g in [21-27]. From [23] we have

Lemma 6.1. Let $A$ be a positive operator in a Banach space $E, b$ be a nonnegative real number and $r=\left(r_{1}, r_{2}, \ldots, r_{n}\right)$ where $r_{k} \in\{0, b\}$. Let $t=\left(t_{1}, t_{2}, \ldots, t_{n}\right), t_{k}$ are positive parameters, $0<t_{k} \leq T<\infty, \alpha=\left(\alpha_{1}, \alpha_{2}, \ldots, \alpha_{n}\right)$ and $l=\left(l_{1}, l_{2}, \ldots, l_{n}\right)$, 
where $l_{k}$ are positive and $\alpha_{k}$ are nonnegative integers such that $\varkappa=|(\alpha+r): l| \leq 1$. For $0<h \leq h_{0}<\infty$ and, $0 \leq \mu \leq 1-\varkappa$ the operator-function

$$
\Psi_{t}(\xi)=\prod_{k=1}^{n} t_{k}^{\frac{\alpha_{k}+r_{k}}{l_{k}}} \xi^{r}(i \xi)^{\alpha} A^{1-\varkappa-\mu} h^{-\mu}[A+\psi(t, \xi)]^{-1}
$$

is bounded operator in $E$ uniformly with respect to $\xi \in \mathbb{R}^{n}, h>0$ and $t$, i.e there is a constant $C_{\mu}$ such that

$$
\left\|\Psi_{t, h, \mu}(\xi)\right\|_{L(E)} \leq C_{\mu}
$$

for all $\xi \in \mathbb{R}^{n}$ and $h>0$, where,

$$
\psi=\psi(t, \xi)=\sum_{k=1}^{n} t_{k}\left|\xi_{k}\right|^{l_{k}}+h^{-1}
$$

Let

$$
\begin{gathered}
\alpha=\left(\alpha_{1}, \alpha_{2}, \ldots, \alpha_{n}\right), l=\left(l_{1}, l_{2}, \ldots, l_{n}\right), \varkappa=\sum_{k=1}^{n} \frac{\alpha_{k}}{l_{k}}, \\
Y=B_{p, \theta, \gamma}^{s}\left(\mathbb{R}^{n} ; E\right), B^{l, s}\left(\mathbb{R}^{n}\right)=B_{p, \theta, \gamma}^{l, s}\left(\mathbb{R}^{n} ; E(A), E\right) .
\end{gathered}
$$

Let $l=\left(l_{1}, l_{2}, \ldots, l_{n}\right)$, where $l_{k}$ are positive integers. Let

$$
\nu(l)=\max _{k,, j \in\{1,2, \ldots, n\}}\left[\frac{1}{l_{k}}-\frac{1}{l_{j}}\right], \eta=\eta(t)=\prod_{k=1}^{n} t_{k}^{\frac{\alpha_{k}}{l_{k}}} .
$$

Theorem 6.1. Suppose that the following conditions hold:

(1) $\gamma \in A_{p}$ for $p \in[1, \infty], E$ is a Banach spaces with weighted Fourier type $\gamma$ and $\sigma \in(1,2]$

(2) $t=\left(t_{1}, t_{2}, \ldots, t_{n}\right), 0<t_{k} \leq T<\infty, 1<p \leq q<\infty, \theta \in[1, \infty]$;

(3) $l_{k}$ are positive and $\alpha_{k}$ are nonnegative integers such that $0<\varkappa+\nu(l) \leq 1$, and let $0 \leq \mu \leq 1-\varkappa-\nu(l)$;

(4) $A$ is a $\varphi$-positive operator in $E$.

Then an embedding

$$
D^{\alpha} B_{p, \theta, \gamma}^{l, s}\left(\mathbb{R}^{n} ; E(A), E\right) \subset B_{p, \theta, \gamma}^{s}\left(\mathbb{R}^{n} ; E\left(A^{1-\varkappa-\mu}\right)\right)
$$

is continuous and there exists a constant $C_{\mu}>0$, depending only on $\mu$, such that the following uniform estimate holds

$$
\eta(t)\left\|D^{\alpha} u\right\|_{B_{p, \theta, \gamma}^{s}\left(\mathbb{R}^{n} ; E\left(A^{1-\varkappa-\mu}\right)\right)} \leq C_{\mu}\left[h^{\mu}\|u\|_{B^{l, s}\left(\mathbb{R}^{n}\right)}+h^{-(1-\mu)}\|u\|_{Y}\right]
$$

for all $u \in B^{l, s}\left(\mathbb{R}^{n}\right)$ and $0<h \leq h_{0}<\infty$.

Proof. We have

$$
\left\|D^{\alpha} u\right\|_{B_{p, \theta, \gamma}^{s}\left(\mathbb{R}^{n} ; E\left(A^{1-\varkappa-\mu}\right)\right)}=\left\|A^{1-\varkappa-\mu} D^{\alpha} u\right\|_{Y}
$$

for all $u$ such that

$$
\left\|D^{\alpha} u\right\|_{B_{p \ddot{\theta} \theta, \gamma}^{s}\left(\mathbb{R}^{n} ; E\left(A^{1-\varkappa-\mu}\right)\right)}<\infty .
$$

On the other hand by using the relation (6.2) we have

$$
A^{1-\alpha-\mu} D^{\alpha} u=F^{-1} F A^{1-\varkappa-\mu} D^{\alpha} u=F^{-1} A^{1-\varkappa-\mu} F D^{\alpha} u
$$




$$
=F^{-1} A^{1-\varkappa-\mu}(i \xi)^{\alpha} F u=F^{-1}(i \xi)^{\alpha} A^{1-\varkappa-\mu} F u .
$$

Hence denoting $F u$ by $\widehat{u}$, we get from the relations (6.2) and (6.3)

$$
\left\|D^{\alpha} u\right\|_{B_{p, \theta, \gamma}^{s}\left(\mathbb{R}^{n} ; E\left(A^{1-\varkappa-\mu}\right)\right)} \backsim\left\|F^{-1}(i \xi)^{\alpha} A^{1-\varkappa-\mu} \widehat{u}\right\|_{Y} .
$$

Similarly, from definition of for all $u \in Y$ we have

$$
\begin{aligned}
& \|u\|_{B^{l, s}\left(\mathbb{R}^{n}\right)}=\|u\|_{B_{p, \theta, \gamma}^{s}\left(\mathbb{R}^{n} ; E(A)\right)}+\sum_{k=1}^{n}\left\|t_{k} D_{k}^{l_{k}} u\right\|_{Y} \\
= & \left\|F^{-1} \widehat{u}\right\|_{B_{p, \theta, \gamma}^{s}\left(\mathbb{R}^{n} ; E(A)\right)}+\sum_{k=1}^{n}\left\|t_{k} F^{-1}\left[\left(i \xi_{k}\right)^{l_{k}} \widehat{u}\right]\right\|_{Y} \\
& \sim\left\|F^{-1} A \widehat{u}\right\|_{Y}+\sum_{k=1}^{n}\left\|t_{k} F^{-1}\left[\left(i \xi_{k}\right)^{l_{k}} \widehat{u}\right]\right\|_{Y} .
\end{aligned}
$$

Thus proving the inequality (6.1) for some constants $C_{\mu}$ is equivalent to proving

$$
\begin{gathered}
\eta\left\|F^{-1}(i \xi)^{\alpha} A^{1-\varkappa-\mu \widehat{u}}\right\|_{B^{l, s}\left(\mathbb{R}^{n}\right)} \\
\left.\leq C_{\mu}\left[h^{\mu}\left(\left\|F^{-1} A \widehat{u}\right\|_{Y}+\sum_{k=1}^{n}\left\|t_{k} F^{-1}\left[\left(i \xi_{k}\right)^{l_{k}} \widehat{u}\right]\right\|_{Y}\right)+h^{-(1-\mu)}\left\|F^{-1} \widehat{u}\right\|_{Y}\right)\right] .
\end{gathered}
$$

Thus the inequality (6.1) will be followed if we prove the following inequality

$$
\eta\left\|F^{-1}\left[(i \xi)^{\alpha} A^{1-\varkappa-\mu} \widehat{u}\right]\right\|_{Y} \leq C_{\mu} \| F^{-1}\left[h^{\mu}(A+\psi(t, \xi)] \widehat{u} \|_{Y}\right.
$$

for a suitable $C_{\mu}>0$ and for all $u \in Y$.

Let us express the left hand side of (6.3) as follows

$$
\begin{gathered}
\eta\left\|F^{-1}\left[(i \xi)^{\alpha} A^{1-\varkappa-\mu} \widehat{u}\right]\right\|_{Y} \\
=\eta \| F^{-1}(i \xi)^{\alpha} A^{1-\varkappa-\mu}\left[h^{\mu}(A+\psi]^{-1}\left[h^{\mu}(A+\psi)\right] \|_{Y} .\right.
\end{gathered}
$$

(Since $A$ is a positive operator in $E$ and $-\psi(t, \xi) \in S(\varphi)$ so it is possible). It is clear that the inequality (6.4) will be followed immediately from (6.5) if we can prove that the operator-function

$$
\Psi_{t}=\Psi_{t, h, \mu}=\eta(t)(i \xi)^{\alpha} A^{1-\varkappa-\mu}\left[h^{\mu}(A+\psi)\right]^{-1}
$$

is a multiplier in $M_{p, \theta, \gamma}^{s, \gamma}(E)$, which is uniformly with respect to $h$ and $t$. In order to prove that $\Psi_{t} \in M_{p, \theta, \gamma}^{s, \gamma}(E)$ it suffices to show that there exists a constant $M_{\mu}>0$ with

$$
|\xi|^{k}\left\|D^{\beta} \Psi_{t}(\xi)\right\|_{L(E)} \leq C, k=0,1, \ldots,|\beta|
$$

for all

$$
\beta=\left(\beta_{1}, \beta_{2}, \ldots, \beta_{n}\right), \beta_{k} \in\{0,1\}, \xi_{k} \neq 0 .
$$

To see this, we apply Lemma 6.1 and get a constant $M_{\mu}>0$ depending only on $\mu$ such that

$$
\left\|\Psi_{t}(\xi)\right\|_{L(E)} \leq M_{\mu}
$$


for all $\xi \in \mathbb{R}^{n}$. This shows that the inequality (7.6) is satisfied for $\beta=(0, \ldots, 0)$. We next consider (6.6) for $\beta=\left(\beta_{1}, \ldots \beta_{n}\right)$ where $\beta_{k}=1$ and $\beta_{k}=0$ for $j \neq k$. By using the condition $\varkappa+\nu(l) \leq 1$ and well known inequality

$$
y_{1}^{\alpha_{1}} y_{2}^{\alpha_{2}} \ldots y_{n}^{\alpha_{n}} \leq C\left[1+\sum_{k=1}^{n} y_{k}^{l_{k}}\right] \text {, for } y_{k} \geq 0
$$

we have

$$
|\xi|\left|\xi_{k}\right|\left\|D_{k} \Psi_{t}(\xi)\right\|_{L(E)} \leq M_{\mu}, k=1,2 \ldots n .
$$

Repeating the above process we obtain the estimate (7.6). Thus the operatorfunction $\Psi_{t, h, \mu}(\xi)$ is a uniform collection of multiplier with respect to $h$ and $t$ i.e

$$
\Psi_{t, h, \mu} \in \Phi_{h} \subset M_{p, \theta, \gamma}^{s, \gamma}(E) .
$$

This completes the proof of the Theorem 6.1. It is possible to state Theorem 6.1 in a more general setting. For this, we use the conception of extension operator.

Let

$$
Y=B_{p, \theta, \gamma}^{s}(\Omega ; E), B^{l, s}(\Omega)=B_{p, \theta, \gamma}^{l, s}(\Omega ; E(A), E) .
$$

Condition 6.1. Let $\gamma \in A_{\nu}$ for $\nu \in[1, \infty]$. Assume that $E$ is a Banach spaces with weighted Fourier type $\gamma$ and $\sigma \in[1,2]$. Suppose $A$ is a $\varphi$-positive operator in Banach spaces $E$. Let a region $\Omega \subset \mathbb{R}^{n}$ be such that there exists a bounded linear extension operator $B$ from $B^{l, s}(\Omega)$ to $B^{l, s}\left(\mathbb{R}^{n}\right)$ for $p, \theta \in[1, \infty]$.

Remark 6.1. If $\Omega \subset \mathbb{R}^{n}$ is a region satisfying a strong $l$-horn condition (see $[4], \S 18) E=\mathbb{C}, A=I$, then there exists a bounded linear extension operator from $B_{p, \theta}^{s}(\Omega)=B_{p, \theta}^{s}(\Omega ; \mathbb{C}, \mathbb{C})$ to

$$
B_{p, \theta}^{s}\left(\mathbb{R}^{n}\right)=B_{p, \theta}^{s}\left(\mathbb{R}^{n} ; \mathbb{C}, \mathbb{C}\right) .
$$

Theorem 6.2. Suppose that the all conditions of the Theorem 6.1 and the Condition 6.1 are hold. Then the embedding

$$
D^{\alpha} B^{l, s}(\Omega) \subset B_{q, \theta, \gamma}^{s}\left(\Omega ; E\left(A^{1-\varkappa-\mu}\right)\right)
$$

is continuous and there exists a constant $C_{\mu}$ depending only on $\mu$ such that

$$
\eta\left\|D^{\alpha} u\right\|_{B_{q, \theta, \gamma}^{s}\left(\Omega ; E\left(A^{1-\varkappa-\mu}\right)\right)} \leq C_{\mu}\left[h^{\mu}\|u\|_{B^{l, s}(\Omega)}+h^{-(1-\mu)}\|u\|_{Y}\right]
$$

for all $u \in B^{l, s}(\Omega)$ and $0<h \leq h_{0}<\infty$.

Proof. It suffices to prove the estimate (7.7). Let $P$ be a bounded linear extension operator from $B_{q, \theta, \gamma}^{s}(\Omega ; E)$ to $B_{q, \theta, \gamma}^{s}\left(\mathbb{R}^{n} ; E\right)$ and also from to $B^{l, s}(\Omega)$ to $B^{l, s}\left(\mathbb{R}^{n}\right)$. Let $P_{\Omega}$ a restriction operator from $\mathbb{R}^{n}$ to $\Omega$. Then for any $u \in Y$ we have

$$
\begin{gathered}
\left\|D^{\alpha} u\right\|_{B_{q, \theta, \gamma}^{s}\left(\Omega ; E\left(A^{1-\varkappa-\mu}\right)\right)} \\
=\left\|D^{\alpha} P_{\Omega} P u\right\|_{B_{q, \theta, \gamma}^{s}\left(\Omega ; E\left(A^{1-\varkappa-\mu}\right)\right)} \leq C\left\|D^{\alpha} P u\right\|_{B_{q, \theta, \gamma}^{s}\left(\mathbb{R}^{n} ; E\left(A^{1-\varkappa-\mu}\right)\right)} \\
\leq C_{\mu}\left[h^{\mu}\|P u\|_{B^{l, s}\left(\mathbb{R}^{n}\right)}+h^{-(1-\mu)}\|P u\|_{B_{p, \theta, \gamma}^{s}\left(\mathbb{R}^{n} ; E\right)}\right] \\
\leq C \mu\left[h^{\mu}\|u\|_{B^{l, s}(\Omega)}+h^{-(1-\mu)}\|u\|_{Y}\right] .
\end{gathered}
$$


Result 6.1. Let the all conditions of Theorem 6.2 hold. Then for all $u \in Y_{0}$ we have the following multiplicative estimate

$$
\left\|D^{\alpha} u\right\|_{B_{q, \theta, \gamma}^{s}\left(\Omega ; E\left(A^{1-\varkappa-\mu}\right)\right)} \leq C_{\mu}\|u\|_{B_{p}^{l, s}(\Omega)}^{1-\mu}\|u\|_{Y}^{\mu} .
$$

Indeed setting $h=\|u\|_{Y} \cdot\|u\|_{B^{l, s}(\Omega)}^{-1}$ in (6.7) we obtain (6.8).

Result 6.2. If $l_{1}=l_{2}=\ldots=l_{n}=m$, then we obtain the continuity of embedding operators in the isotropic class

$$
B_{p, \theta, \gamma}^{m, s}(\Omega ; E(A) E) .
$$

For $E=\mathbb{C}, A=I$ we obtain the embedding of weighted Besov type spaces

$$
D^{\alpha} B_{p, \theta, \gamma}^{l, s}(\Omega) \subset B_{q, \theta, \gamma}^{s}(\Omega) .
$$

\section{$7 \mathbb{B}$-separable abstract differential equation on $\mathbb{R}^{n}$}

Let us consider the equation (1.1).

Condition 7.1. Let

(a) $K(\xi)=\sum_{|\alpha: l|=1} a_{\alpha}\left(i \xi_{1}\right)^{\alpha_{1}}\left(i \xi_{2}\right)^{\alpha_{2}} \ldots\left(i \xi_{n}\right)^{\alpha_{n}} \in S(\varphi), \varphi<\frac{\pi}{2}$;

(b) There exists a positive constat $M_{0}$ so that

$$
|K(\xi)| \geq M_{0} \sum_{k=1}^{n}\left|\xi_{k}\right|^{l_{k}} \text { for all } \xi \in \mathbb{R}^{n}, \xi \neq 0 .
$$

Consider the following degenerate abstract differential equation

$$
L u=\sum_{|\alpha: l|=1} a_{\alpha} D^{[\alpha]} u+A u+\sum_{|\alpha: l|<1} A_{\alpha} D^{[\alpha]} u=f,
$$

where $A, A_{\alpha}(x)$ are linear operators in a Banach space $E, a_{k}$ are complex-valued functions and

$$
D_{x_{k}}^{[i]}=\left(\gamma\left(x_{k}\right) \frac{\partial}{\partial x_{k}}\right)^{i}, D^{[\alpha]}=D_{1}^{\left[\alpha_{1}\right]} D_{2}^{\left[\alpha_{2}\right]} \ldots D_{n}^{\left[\alpha_{n}\right]}
$$

Here, $B_{q, \theta, \gamma}^{[l], s}\left(\Omega ; E_{0}, E\right)$ denote a $E$-valued Sobolev-Besov weighted space of functions $u \in B_{q, \theta}^{s}\left(\mathbb{R}^{n} ; E\right)$ that have weak derivatives $D_{k}^{\left[l_{k}\right]} u \in B_{q, \theta}^{s}\left(\mathbb{R}^{n} ; E\right)$ with the norm

$$
\|u\|_{B_{q, \theta}^{[l,], s}\left(\mathbb{R}^{n} ; E_{0}, E\right)}=\|u\|_{B_{q, \theta}^{s}\left(\mathbb{R}^{n} ; E_{0}\right)}+\sum_{k=1}^{n}\left\|D_{k}^{\left[l_{k}\right]} u\right\|_{B_{q, \theta}^{s}\left(\mathbb{R}^{n} ; E\right)}<\infty .
$$

Remark 7.1. Under the substitution

$$
\tau_{k}=\int_{0}^{x_{k}} \gamma^{-1}(y) d y
$$


the spaces $B_{p, \theta, \gamma}^{s}\left(\mathbb{R}^{n} ; E\right), B_{p, \theta, \gamma}^{[l], s}\left(\mathbb{R}^{n} ; E(A), E\right)$ are mapped isomorphically onto the weighted spaces $B_{p, \theta, \tilde{\gamma}}^{s}\left(\mathbb{R}^{n} ; E\right), B_{p, \theta, \tilde{\gamma}}^{l, s}\left(\mathbb{R}^{n} ; E(A), E\right)$, respectively, where

$$
\gamma=\prod_{k=1}^{n} \gamma\left(x_{k}\right), \tilde{\gamma}=\tilde{\gamma}(\tau)=\prod_{k=1}^{n} \gamma\left(x_{k}\left(\tau_{k}\right)\right)
$$

Moreover, under the substitution (7.2) the degenerate problem (7.1) is mapped to the nondegenerate problem (1.1) considered in the weighted space $B_{p, \theta, \tilde{\gamma}}^{s}\left(\mathbb{R}^{n} ; E\right)$.

Let

$$
Y=B_{q, \theta, \gamma}^{s}\left(\mathbb{R}^{n} ; E\right), Y_{0}=B_{q, \theta, \gamma}^{l, s}\left(\mathbb{R}^{n} ; E(A), E\right) .
$$

Theorem 7.1. Suppose that the following conditions hold:

(1) Condition 7.1 is hold, $s>0,1 \leq q, \theta \leq \infty$ and $0<\mu<1-|\alpha: l|$;

(2) $\gamma \in A_{q}$ for $q \in[1, \infty]$. $E$ is a Banach spaces with weighted Fourier type $\gamma$ and $p \in[1,2]$;

(4) $A$ is a $\varphi$-positive operator in $E$ and

$$
A_{\alpha}(x) A^{-(1-|\alpha: l|-\mu)} \in L_{\infty}\left(\mathbb{R}^{n} ; L(E)\right) .
$$

Then for all $f \in Y$ and for sufficiently large $|\lambda|, \lambda \in S(\varphi)$ equation (1.1) has a unique solution $u(x) \in Y_{0}$ and

$$
\sum_{|\alpha: l|=1}\left\|D^{\alpha} u\right\|_{Y}+\|A u\|_{Y} \leq C\|f\|_{Y} .
$$

Proof. Firstly, we will consider the leading part of (1.1) i.e. consider the differentialoperator equation

$$
\left(L_{0}+\lambda\right) u=\sum_{|\alpha: l|=1} D^{\alpha} u+A u+\lambda u=f .
$$

Then we apply the Fourier transform to equation (7.4) with respect to $x=\left(x_{1}, \ldots, x_{n}\right)$ and obtain

$$
K(\xi) \widehat{u}(\xi)+(A+\lambda) \widehat{u}(\xi)=\widehat{f}(\xi) .
$$

Since $K(\xi) \in S(\varphi)$ for all $\xi \in \mathbb{R}^{n}$ therefore, $\omega=\omega(\lambda, \xi)=\lambda+K(\xi) \in S(\varphi)$ for all $\xi \in \mathbb{R}^{n}$, i.e. operator $A+\omega$ is invertible in $E$. Hence (7.5) implies that the solution of equation (7.4) can be represented in the form

$$
u(x)=F^{-1}(A+\omega)^{-1} \widehat{f} .
$$

It is clear to see that the operator- function $\varphi_{\lambda}(\xi)=[A+\omega]^{-1}$ is a multiplier in $Y$ uniformly with respect to $\lambda$. Actually, by definition of the positive operator, for all $\xi \in \mathbb{R}^{n}$ and $\lambda \geq 0$ we get

$$
\left\|\varphi_{\lambda}(\xi)\right\|_{L(E)}=\left\|(A+\omega)^{-1}\right\| \leq M(1+|\omega|)^{-1} \leq M_{0} .
$$

Moreover, since $D_{k} \varphi_{\lambda}(\xi)=\alpha_{k} a_{\alpha} \xi^{\alpha}(A+\omega)^{-2} \xi_{k}^{-1}$, then by using the resolvent properties of positive operator $A$ we have

$$
\left\|\xi_{k} D_{k} \varphi_{\lambda}\right\|_{L(E)} \leq\left|\alpha_{k} a_{\alpha}\right| \xi^{\alpha}\left\|(A+\omega I)^{-2}\right\| \leq M .
$$


Using the estimate (7.7) we show the uniform estimate

$$
|\xi|^{\beta}\left\|D_{\xi}^{\beta} \varphi_{\lambda}(\xi)\right\|_{L(E)} \leq C
$$

for

$$
\left.\beta=\beta_{1}, \ldots, \beta_{n}\right), \beta_{i} \in\{0,1\}, \xi=\left(\xi_{1}, \ldots, \xi_{n}\right), \xi_{i} \neq 0 .
$$

In a similar way we can prove that the operator-functions $\varphi_{\alpha \lambda}(\xi)=\xi^{\alpha} \varphi_{\lambda, t}, k=$ $1,2, . ., n$ and $\varphi_{0 \lambda}=A \varphi_{\lambda}$ satisfiy the estimates

$$
(1+|\xi|)^{|\beta|}\left\|D_{\xi}^{\beta} \varphi_{\alpha, \lambda}(\xi)\right\|_{L(E)} \leq C,(1+|\xi|)^{|\beta|}\left\|D_{\xi}^{\beta} \varphi_{0, \lambda}(\xi)\right\|_{L(E)} \leq C .
$$

Then in view of (7.8) and (7.9) we obtain that operator-functions $\varphi_{\lambda}, \varphi_{\alpha \lambda}, \varphi_{0, \lambda}$ are multipliers in $Y$. By (7.9) and in view of

$$
\begin{gathered}
\left\|D^{\alpha} u\right\|_{Y}=\left\|F^{-1} \xi^{\alpha} \widehat{u}\right\|_{Y}=\left\|F^{-1} \xi^{\alpha}(A+\omega)^{-1} \widehat{f}\right\|_{Y}, \\
\|A u\|_{Y}=\left\|F^{-1} A \widehat{u}\right\|_{Y}=\left\|F^{-1}\left[A(A+\omega)^{-1}\right] \widehat{f}\right\|_{Y} .
\end{gathered}
$$

we obtain that there exists a unique solution of equation (7.4) for all $f \in Y$ and the uniform estimate holds

$$
\sum_{|\alpha: l|=1}\left\|D^{\alpha} u\right\|_{Y}+\|A u\|_{Y} \leq C\|f\|_{Y} .
$$

We consider the differential operator $G_{0}$ generated by problem (7.4), that is

$$
D\left(G_{0}\right)=Y_{0}, G_{0} u=\sum_{|\alpha: l|=1} D^{\alpha} u+A u .
$$

The estimate (7.10) implies that the operator $G_{0}+\lambda$ has a bounded inverse from $Y$ into $Y_{0}$ for all $\lambda \geq 0$. Let $G$ denote the differential operator in $Y$ generated by problem (1.1). Namely,

$$
D(G)=Y_{0}, G u=G_{0} u+L_{1} u, \quad L_{1} u=\sum_{|\alpha: l|<1} A_{\alpha}(x) D^{\alpha} u .
$$

In view of (4) condition, by virtue of Theorem 6.1 , for all $u \in Y$ we have

$$
\begin{gathered}
\left\|L_{1} u\right\|_{Y} \leq \sum_{|\alpha: l|<1}\left\|A_{\alpha}(x) D^{\alpha} u\right\|_{Y} \leq \sum_{|\alpha: l|<1}\left\|A^{1-|\alpha: l|-\mu} D^{\alpha} u\right\|_{Y} \\
\leq C\left[h^{\mu}\left(\sum_{|\alpha: l|=1}\left\|D^{\alpha} u\right\|_{Y}+\|A u\|_{Y}\right)+h^{-(1-\mu)}\|u\|_{Y}\right] .
\end{gathered}
$$

Then from estimates (7.10) and (7.12) for $u \in Y_{0}$ we obtain

$$
\left\|L_{1} u\right\|_{Y} \leq C\left[h^{\mu}\left\|\left(G_{0}+\lambda\right) u\right\|_{Y}+h^{-(1-\mu)}\|u\|_{Y}\right] .
$$

Since $\|u\|_{Y}=\frac{1}{\lambda}\left\|\left(G_{0}+\lambda\right) u-G_{0} u\right\|_{Y}$ for all $u \in Y_{0}$ we get

$$
\|u\|_{Y} \leq \frac{1}{|\lambda|}\left[\left\|\left(G_{0}+\lambda\right) u\right\|_{Y}+\left\|G_{0 t} u\right\|_{Y}\right],
$$




$$
\left\|G_{0} u\right\|_{Y} \leq C\left[\sum_{|\alpha|=l}\left\|D^{\alpha} u\right\|_{B_{p, \theta, \gamma}^{s}}+\|A u\|_{B_{p, \theta, \gamma}^{s}}\right] .
$$

From estimates $(7.12)-(7.14)$ for all $u \in Y_{0}$ we obtain

$$
\left\|L_{1} u\right\|_{Y} \leq C h^{\mu}\left\|\left(G_{0}+\lambda\right) u\right\|_{Y}+C_{1}|\lambda|^{-1} h^{-(1-\mu)}\left\|\left(G_{0}+\lambda\right) u\right\|_{Y} .
$$

Then by choosing $h$ and $\lambda$ such that $C h^{\mu}<1, C_{1}|\lambda|^{-1} h^{-(1-\mu)}<1$ from (7.15) we obtain the uniform estimate

$$
\left\|L_{1}\left(G_{0}+\lambda\right)^{-1}\right\|_{L(E)}<1 .
$$

Using the relation (7.11), estimates (7.10) and (7.16) and the perturbation theory of linear operators we obtain that the differential operator $G+\lambda$ is invertible from $Y$ into $Y_{0}$. Hence, inequality (7.3) is valid and this complete the proof.

Result 7.1. The Theorem 8.1 implies that $G$ has a resolvent operator $(G+\lambda)^{-1}$ for $|\arg \lambda| \leq \varphi$ and the following uniform estimate holds

$$
\sum_{|\alpha: l| \leq 1}|\lambda|^{1-|\alpha: l|}\left\|D^{\alpha}(G+\lambda)^{-1}\right\|_{L(Y)}+\left\|A(G+\lambda)^{-1}\right\|_{L(Y)} \leq C .
$$

Let

$$
Y=B_{q, \theta}^{s}\left(\mathbb{R}^{n} ; E\right), Y_{0}=B_{q, \theta, \gamma}^{[l], s}\left(\mathbb{R}^{n} ; E(A), E\right) .
$$

Let $Q$ denote the operator in $B_{q, \theta}^{s}\left(\mathbb{R}^{n}, E\right)$ generated by problem (7.1). Theorem 7.1 and Remark 7.1 imply

Result 7.2. Let all conditions of Theorem 7.1 hold. Then for all $f \in Y, \lambda \in S(\varphi)$

and for sufficiently large $|\lambda|$, the equation (7.1) has a unique solution $u \in Y_{0}$ and the coercive uniform estimate holds

$$
\sum_{|\alpha: l| \leq 1}|\lambda|^{1-|\alpha: l|}\left\|D^{[\alpha]}(Q+\lambda)^{-1}\right\|_{L(Y)}+\left\|A(Q+\lambda)^{-1}\right\|_{L(Y)} \leq C .
$$

Remark 7.2. The Result 7.2 implies that $G$ is a positive operator in $Y$. Then by virtue of $[28, \S 1.14 .5]$ the operator $G$ is a generator of an analytic semigroup in $Y$ for $\varphi \in\left(\frac{\pi}{2}, \pi\right)$.

\section{References}

1. Amann, H.: Linear and quasi-linear equations,1, Birkhauser, Basel, 1995.

2. Amann, H.: Operator-valued Fourier multipliers, vector-valued Besov spaces, and applications, Math. Nachr. 186, 5-56 (1997).

3. Agarwal, R., O' Regan, D., Shakhmurov V.B.: Separable anisotropic differential operators in weighted abstract spaces and applications, J. Math. Anal. Appl. 338, 970-983 (2008).

4. Besov, O.V., Ilin, V.P., Nikolskii, S.M.: Integral representations of functions and embedding theorems, Nauka, Moscow, 1975. 
5. Bergh J., Lofstrom J.: Interpolation spaces, An introduction, Springer-Verlag, Berlin, Grundlehren der Mathematischen Wissenschaften, no. 223. (1976).

6. Bourgain, J.: A Hausdorff - Young inequality for B-convex banach spaces, Pacific J. Math. 101 (2), 255-262 (1982).

7. Diestel, J., Jarchow H., Tonge A.: Absolutely summing operators, Cambridge Univ. Press, Cambridge, 1995.

8. Denk, R., Hieber M., Prüss J.: $R$ - boundedness, Fourier multipliers and problems of elliptic and parabolic type, Mem. Amer. Math. Soc. 166(788), 114 pp. (2003).

9. Fackler, S., Hytönen, T.P., Lindemulder, N.: Weighted estimates for operatorvalued Fourier multipliers, Collect. Math. 71, 511-548 (2020).

10. Girardi, M., Lutz, W.: Operator-valued Fourier multiplier theorems on Besov spaces, Math. Nachr. 251, 34-51 (2003).

11. Guliyev, V.S.: To the theory of multiplicators of Fourier integrals for banachvalued functions, Trud. Math. Inst. Steklov, 214, 157-174 (1996).

12. Guliyev, V.S., Omarova, M.N., Ragusa, M.A., Scapellato, A.: Commutators and generalized local Morrey spaces, J. Math. Anal. Appl. 457(2), 1388-1402 (2018).

13. Haller, R., Heck, H., Noll A.: Mikhlin's theorem for operator-valued Fourier multipliers in $n$ variables, Math. Nachr. 244, 110-130 (2002).

14. Kurtz, D.G., Whedeen, R.L.: Results on weighted norm inequalities for multipliers, Trans. Amer. Math. Soc. 255, 343-362 (1979).

15. Lions, J.L., Peetre, J.: Sur une classe d'espaces d'interpolation, Inst. Hautes Etudes Sci. Publ. Math. 19, 5-68 (1964).

16. Meyries, M., Veraar, M.: Pointwise multiplication on vector-valued function spaces with power weights, J. Fourier Anal. Appl. 21(1), 95-136 (2015).

17. McConnell Terry R.: On Fourier Multiplier Transformations of banach-valued Functions, Trans. Amer. Mat. Soc. 285(2), 739-757 (2015).

18. Muckenhoupt, B.: Hardy's inequality with weights, Studia Math. 44(1), 31-38 (1972).

19. Peetre J.: Sur la transformation de Fourier des fonctions a valeurs vectorielles, Rend. Sem. Mat. Univ. Padova 42, 15-26 (1969).

20. Stein, E.M.: Singular integrals and differentiability properties of functions, Princeton University Press (1970).

21. Shakhmurov, V.B., Jebrailov, M.S.: About compactness of an embedding Bspaces, Rep. Acad. Sci. Azerb. SSR. (3), 1990.

22. Shakhmurov, V.B.: Imbedding theorems and their applications to degenerate equations, Differ. Equ. 24(4), 475-482 (1988).

23. Shakhmurov, V.B.: Embedding operators and maximal regular differentialoperator equations in banach-valued function spaces, J. Inequal. Appl. 4, 329-345 (2005).

24. Shakhmurov, V.B.: Linear and nonlinear abstract equations with parameters, Nonlinear Anal. 73, 2383-2397 (2010).

25. Shakhmurov, V.B.: Coercive boundary value problems for regular degenerate differential-operator equations, J. Math. Anal. Appl. 292(2), 605-620 (2004).

26. Shakhmurov, V.B.: Embedding and maximal regular differential operators in Sobolev-Lions spaces, Acta Math. Sin. (Engl. Ser.) 22(5), 1493-1508 (2006).

27. Schmeisser H.: Vector-valued Sobolev and Besov spaces, Seminar analysis of the Karl-Weierstra-Institute of Mathematics 1985/86 (Berlin, 1985/86), Teubner, Leipzig, 4-44 (1987).

28. Triebel, H.: Interpolation theory. Function spaces. Differential operators, NorthHolland, Amsterdam, 1978. 
29. Triebel, H.: Spaces of distributions with weights. Multipliers in $L_{p}$-spaces with weights, Math. Nachr. 78, 339-356 (1977).

30. Yakubov, S., Yakubov Ya.: Differential-operator equations, Ordinary and Partial Differential equations, Chapman and Hall /CRC, Boca Raton, 2000.

31. Zimmerman, F.: On vector-valued Fourier multiplier theorems, Studia Math. 93(3), 201-222 (1989). 